\title{
Association of methylenetetrahydrofolate reductase C677T and A1298C polymorphisms with colorectal cancer risk: A meta-analysis
}

\author{
MENGMENG ZHAO ${ }^{1,2}$, XUELIAN LI $^{1,2}$, CHENGZHONG XING $^{3}$ and BAOSEN ZHOU ${ }^{1,2}$ \\ ${ }^{1}$ Department of Epidemiology, School of Public Health, China Medical University; \\ ${ }^{2}$ Key Laboratory of Cancer Etiology and Intervention, University of Liaoning Province; \\ ${ }^{3}$ Department of Anorectal Surgery, The First Affiliated Hospital of China Medical University, \\ Shenyang, Liaoning 110001, P.R. China
}

Received April 19, 2013; Accepted July 08, 2013

DOI: $10.3892 /$ br.2013.134

\begin{abstract}
Colorectal cancer (CRC) is one of the most common types of cancer worldwide and a leading cause of cancer-related mortality. This meta-analysis was conducted to determine the effect of methylenetetrahydrofolate reductase (MTHFR) mutants on the risk of CRC. A literature search was conducted on PubMed, Medline and the China National Knowledge Infrastructure (CNKI) databases. Eligible studies were collected based on rigorous criteria of inclusion. The pooled odds ratios (ORs) with $95 \%$ confidence intervals (CIs) were calculated by the fixed- or random-effects model. After all the studies were pooled, the OR of CRC for individuals carrying the MTHFR 677TT genotype, compared to the CC genotype, was 0.89 (95\% CI: 0.82-0.97). When analyzed by ethnicity, Asians with the MTHFR 1298CC genotype exhibited a decreased risk of $\mathrm{CRC}(\mathrm{OR}=0.69 ; 95 \% \mathrm{CI}$ : 0.54-0.89). In a mixed population, a significantly reduced risk of CRC was observed among carriers of the 677TT $(\mathrm{OR}=0.86$; 95\% CI: 0.76-0.96) and the $1298 \mathrm{CC}(\mathrm{OR}=0.82$; 95\% CI: 0.69-0.98) genotypes, compared to the wild-type homozygous genotype. In the subgroup of colon cancer, the OR of 677TT vs. CC+CT was 0.83 (95\% CI: 0.72-0.96) and the OR of $1298 \mathrm{CC}$ vs. AA+AC was 0.81 (95\% CI: 0.69-0.96). In the rectal cancer subgroup, the OR of 677TT vs. CC+CT was 0.86 (95\% CI: 0.77-0.97). Therefore, this meta-analysis suggested that the MTHFR 677T and $1298 \mathrm{C}$ alleles were associated with a low risk of CRC.
\end{abstract}

Correspondence to: Professor Baosen Zhou, Department of Epidemiology, School of Public Health, China Medical University, 92 Beier Road, Heping District, Shenyang, Liaoning 110001, P.R. China

E-mail: bszhou@mail.cmu.edu.cn

Key words: methylenetetrahydrofolate reductase, polymorphism, colorectal cancer, meta-analysis

\section{Introduction}

Colorectal cancer (CRC) is one of the most common types of cancer worldwide (1). It is the fourth leading cause of cancer-related mortality among males and the third among females. Furthermore, the mortality rate of CRC in developed countries was shown to be higher compared to that in developing countries (3).

Folate is a type of water-soluble B vitamin and an essential nutrient required for human metabolism. Folate plays a key role in the formation of S-adenosylmethionine, which is the universal methyl donor for DNA methylation, as well as in the formation of purine and thymidine for DNA synthesis (4). Folate deficiency increases the risk of tumorigenesis through one of the following mechanisms: by leading to aberrant DNA methylation, which may in turn lead to an altered expression of critical tumor suppressor genes and proto-oncogenes; or by causing imbalances in the pools of nucleotide precursors, leading to DNA strand breaks and mutations and disruption of DNA integrity and repair $(4,5)$.

The methylenetetrahydrofolate reductase (MTHFR) is an enzyme that is crucial in the metabolism of folate (6). MTHFR catalyzes the irreversible conversion of 5,10-methylenetetrahydrofolate to 5-methyltetrahydrofolate, the primary methyl donor for the remethylation of homocysteine to methionine. The gene encoding MTHFR is located on chromosome 1p36.3 (7). Two common single-nucleotide polymorphisms of MTHFR are MTHFR C677T (Ala222Val, rs1801133) and A1298C (Glu429Ala, rs1801131). The C677T variant enhances enzyme thermolability and is associated with decreased activity of the MTHFR enzyme (8). The A1298C variant (Glu429Ala, rs1801131) is a missense mutation leading to reduced MTHFR enzyme activity $(9,10)$. The homozygous genotypes of MTHFR C677T and A1298C are associated with higher homocysteine levels, which may lead to DNA hypomethylation and increased cancer prevalence. However, the decreasing enzyme activity results in higher 5,10-methylenetetrahydrofolate and thymidine levels and, thus, increased DNA synthesis and repair. Therefore, MTHFR polymorphisms are regarded as a protective factor against tumor development $(8,11)$. 
It was first reported by Chen et al (10) that the homozygote of the MTHFR 677T allele was associated with a reduced CRC risk. Previous studies reported that the MTHFR 677T or $1298 \mathrm{C}$ allele exerted a protective effect compared to the wild-type genotypes $(12,13)$. However, other studies reported opposing results, i.e., that mutant genotypes were associated with an increased risk of developing CRC $(14,15)$.

A meta-analysis of all published studies was conducted to determine the effect of MTHFR mutants on CRC risk. In a subgroup analysis, the study subjects were classified by ethnicity and tumor location to provide comprehensive evidence on the association of MTHFR C677T and A1298C with CRC.

\section{Materials and methods}

Study identification and selection. A literature search was conducted on PubMed, Medline and China National Knowledge Infrastructure (January, 1991-September, 2012) databases, using the following keywords and subject terms: 'MTHFR', 'polymorphism' and 'colon cancer' or 'rectal cancer'. All the studies in our meta-analysis were required to meet the following inclusion criteria: i) case-control studies; ii) raw data to calculate odds ratios (ORs) with $95 \%$ confidence intervals (95\% CIs); iii) in case of the same results published in multiple studies, the most recent publication or the largest sample was considered. A given study was excluded from this meta-analysis when: i) the genotype or allele frequencies were not reported, ii) the study design was not case-control, iii) the association between MTHFR polymorphisms and colorectal adenoma was investigated.

Data extraction. Data were carefully and independently collected according to the genotypes MTHFR C677T or A1298C. Two authors extracted the following information from the eligible studies: first author's name, publication year, country, ethnicity of participants and number of cases and controls. In our study, ethnicities were classified as European and American, Asian, African and mixed.

Statistical analysis. The association between MTHFR C677T and A1298C gene polymorphisms and CRC risk was assessed by using the codominant (677CT vs. CC; 677TT vs. CC; $1298 \mathrm{AC}$ vs. AA; $1298 \mathrm{CC}$ vs. AA), the dominant (677CT+TT vs. CC; $1298 \mathrm{AC}+\mathrm{CC}$ vs. AA) and the recessive (677TT vs. CC+CT; 1298CC vs. AA+AC) models. The same procedures were applied for the MTHFR A1298C genotype. Subgroup analyses were performed by tumor location and ethnicity of the control groups.

The strength of association of the MTHFR gene polymorphisms with CRC was measured by the ORs (1) together with the $95 \%$ CIs. The significance of the pooled ORs was determined by the Z-test and $\mathrm{P}<0.05$ was considered to indicate a statistically significant difference. The Chi-square test was first used to assess whether the distribution of genotypes among controls conformed to the Hardy-Weinberg equilibrium (HWE), with $\mathrm{P}<0.05$ considered a departure from $\mathrm{HWE}$. The Q-test was used to assess heterogeneity among the studies. When $\mathrm{P}<0.05$, the heterogeneity was considered to indicate a statistically significant difference. The $\mathrm{I}^{2}$ index was used to quantify the percentage of the total variation among studies when heterogeneity was calculated. The $\mathrm{I}^{2}$ value ranged from 0 to $100 \%$, with 25,50 and $75 \%$ expressing low, moderate and high heterogeneity, respectively. When $\mathrm{I}^{2}<50 \%$, a fixed-effects model (the Mantel-Haenszel method) was applied to estimate the pooled results. Otherwise, the random-effects model (the DerSimonian-Laird method) was used.

Publication bias was visually investigated in a funnel plot of $\log (\mathrm{OR})$ against its standard error (SE). An asymmetric plot suggested possible publication bias. The degree of asymmetry was assessed via the Egger's test $(\mathrm{P}<0.05$ was considered publication bias). A sensitivity analysis was performed by omitting each study in turn to assess the stability of the results.

All the analyses were performed with Stata software version 11.0 (StataCorp, College Station, TX, USA). All the P-values were two-sided.

\section{Results}

Selection of studies. A total of 302 studies were identified during the literature search and 232 were excluded due to departures from the inclusion criteria. Eventually, 70 studies were included $(1,2,6,10,12-77)$. One study, conducted by Lee et al (41), consisted of three individual case-control studies and was handled as three populations; furthermore, the studies by Keku et al (12) and Lima et al (28), included two populations each. This meta-analysis included a larger population compared to previous meta-analyses. Of the 74 case-control studies included in the 70 publications, 74 studies investigated MTHFR C677T (29,783 cases and 41,772 controls) and 39 investigated MTHFR A1298C (13,285 cases and 20,164 controls). In total, 33 populations were from Europe and America; 31 were from Asia; 4 were from Africa and the remaining were mixed populations. In addition, a subanalysis was conducted by tumor location. Twenty-one of the 74 studies provided detailed data on colon and 14 on rectal cancer. The characteristics of the studies are listed in Table I.

Meta-analysis. The results of the meta-analysis are presented in Table II. There was obvious heterogeneity for all the models, except for the MTHFR A1298C additive model (CA vs. AA, $\mathrm{P}=0.307, \mathrm{I}^{2}=9.2 \%$ ) and the random-effects model was used to estimate the pooled data. The meta-analysis of the 74 populations demonstrated that MTHFR C677T was associated with reduced CRC risk under the homozygote (TT vs. CC: $\mathrm{OR}=0.89 ; 95 \% \mathrm{CI}: 0.82-0.97 ; \mathrm{P}=0.009$ ) (Fig. 1) and recessive (TT vs. $\mathrm{CC}+\mathrm{CT}$ : $\mathrm{OR}=0.89 ; 95 \% \mathrm{CI}: 0.82-0.96 ; \mathrm{P}=0.003$ ) models (Fig. 2). However, the MTHFR 1298C allele was not associated with a significantly decreased risk of CRC.

The association of MTHFR C677T and A1298C polymorphisms with CRC was further stratified by ethnicity. As shown in Table II, no significant association was observed between MTHFR C677T and the risk of CRC under any genetic models, in any of the populations. However, Asians carrying the MTHFR 1298CC genotype exhibited a reduced risk of CRC. The OR of CC vs. AA was 0.69 (95\% CI: 0.54-0.89) and the OR of CC vs. AA+AC was 0.69 (95\% CI: 0.54-0.88). In the mixed populations, the pooled analysis demonstrated that carriers of MTHFR 677TT and 1298CC were more common among CRC patients than among controls when compared to individuals with wild-type genotypes (677TT vs. CC: $\mathrm{OR}=0.86$; 
Table I. Characteristics of the studies included in the meta-analysis.

MTHFR 677C/T (case/control)

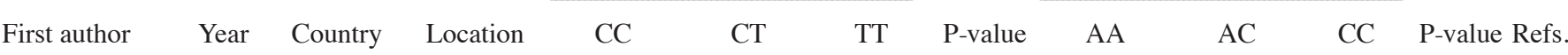

Europe/America

\begin{tabular}{|c|c|c|c|c|c|c|c|c|c|c|c|c|}
\hline Chen et al & 1996 & USA & NR & $67 / 280$ & $64 / 263$ & $13 / 84$ & 0.08 & & & & & 10 \\
\hline Ma et al & 1997 & USA & NR & $92 / 145$ & $92 / 132$ & $18 / 49$ & 0.04 & & & & & 16 \\
\hline Ryan et al & 2001 & Ireland & NR & $49 / 439$ & $73 / 326$ & $14 / 83$ & 0.05 & & & & & 17 \\
\hline Keku et al & 2002 & USA & Colon & $144 / 265$ & $140 / 223$ & $24 / 51$ & 0.68 & $156 / 237$ & $132 / 236$ & $21 / 68$ & 0.44 & 12 \\
\hline Shannon et al & 2002 & Australia & Colon & $249 / 533$ & $197 / 560$ & $55 / 114$ & 0.05 & & & & & 14 \\
\hline Sachse et al & 2002 & UK & NR & $238 / 271$ & $199 / 272$ & $53 / 49$ & 0.09 & & & & & 18 \\
\hline Heijmans et al & 2003 & Netherlands & NR & 7/399 & 7/329 & $4 / 65$ & 0.81 & & & & & 13 \\
\hline Pufulete et al & 2003 & UK & NR & $36 / 41$ & $19 / 29$ & $8 / 6$ & 0.78 & $34 / 47$ & $22 / 26$ & $7 / 3$ & 0.80 & 19 \\
\hline Plaschke et al & 2003 & Germany & NR & $133 / 149$ & $120 / 159$ & $34 / 38$ & 0.65 & $134 / 154$ & $124 / 151$ & $29 / 41$ & 0.67 & 20 \\
\hline Toffoli et al & 2003 & Italy & Colon & $93 / 83$ & $145 / 140$ & $38 / 56$ & 0.83 & $122 / 133$ & $129 / 121$ & $25 / 25$ & 0.74 & 21 \\
\hline Ulvik et al & 2004 & Norway & NR & $1,103 / 1,092$ & 899/886 & $157 / 212$ & 0.10 & & & & & 22 \\
\hline Landi et al & 2005 & Spain & NR & $128 / 109$ & $158 / 139$ & $64 / 61$ & 0.17 & $189 / 170$ & $146 / 127$ & $25 / 22$ & 0.79 & 23 \\
\hline Koushik et al & 2006 & USA & NR & $166 / 355$ & $145 / 327$ & $38 / 112$ & 0.01 & $154 / 389$ & $166 / 332$ & $33 / 85$ & 0.26 & 24 \\
\hline $\begin{array}{l}\text { Van Guelpen } \\
\text { et al }\end{array}$ & 2006 & Sweden & NR & $123 / 212$ & $85 / 161$ & $12 / 42$ & 0.17 & $85 / 189$ & $103 / 173$ & $32 / 50$ & 0.29 & 25 \\
\hline Battistelli et al & 2006 & Italy & NR & $32 / 30$ & $40 / 51$ & $21 / 19$ & 0.75 & & & & & 26 \\
\hline Osian et al & 2007 & Romania & NR & $38 / 47$ & $25 / 17$ & $6 / 3$ & 0.38 & $33 / 41$ & $32 / 25$ & $4 / 1$ & 0.19 & 27 \\
\hline Lima et al & 2007 & Brazil & NR & $36 / 143$ & $46 / 127$ & $15 / 30$ & 0.82 & $68 / 191$ & $28 / 93$ & $6 / 16$ & 0.30 & 28 \\
\hline $\begin{array}{l}\text { Theodoratou } \\
\text { et al }\end{array}$ & 2008 & Scotland & NR & $447 / 439$ & $441 / 455$ & $111 / 116$ & 0.91 & $465 / 462$ & $425 / 445$ & $106 / 102$ & 0.73 & 29 \\
\hline Sharp et al & 2008 & Scotland & NR & $17 / 170$ & $111 / 177$ & $23 / 47$ & 0.93 & $105 / 177$ & $111 / 157$ & $29 / 60$ & 0.01 & 30 \\
\hline Eklöf et al & 2008 & Sweden & NR & & & & 0.15 & & & & & 31 \\
\hline Küry et al & 2008 & France & NR & $435 / 457$ & $452 / 515$ & $136 / 149$ & 0.84 & $484 / 577$ & $432 / 443$ & $107 / 101$ & 0.23 & 32 \\
\hline Derwinger et al & 2009 & Sweden & NR & $273 / 167$ & $216 / 107$ & $55 / 25$ & 0.19 & & & & & 33 \\
\hline $\begin{array}{l}\text { de Vogel et al } \\
\text { Gallegos- }\end{array}$ & 2009 & Netherlands & NR & $318 / 876$ & $320 / 750$ & $51 / 167$ & 0.72 & $299 / 735$ & $275 / 774$ & $110 / 258$ & 0.02 & 34 \\
\hline $\begin{array}{l}\text { Arreola et al } \\
\text { Fernández- }\end{array}$ & 2009 & Mexico & NR & $124 / 59$ & $126 / 79$ & $119 / 32$ & 0.54 & & & & & 36 \\
\hline Peralta et al & 2010 & Spain & NR & $89 / 44$ & $52 / 50$ & $2 / 9$ & 0.32 & $84 / 57$ & $53 / 44$ & $6 / 2$ & 0.05 & 35 \\
\hline Komlosi et al & 2010 & Hungary & $\begin{array}{l}\text { Colon } \\
\text { Rectum }\end{array}$ & $\begin{array}{l}208 / 216 \\
190 / 226\end{array}$ & $\begin{array}{l}196 / 186 \\
231 / 194\end{array}$ & $\begin{array}{l}68 / 59 \\
58 / 58\end{array}$ & 0.01 & & & & & 37 \\
\hline Karpinski et al & 2010 & Poland & NR & $74 / 71$ & $97 / 55$ & $15 / 14$ & 0.49 & & & & & 38 \\
\hline Eussen et al & 2010 & Europe & NR & $567 / 1,019$ & $608 / 1,076$ & $154 / 271$ & 0.61 & $605 / 1,099$ & $574 / 1,007$ & $151 / 259$ & 0.22 & 40 \\
\hline Pardini et al & 2011 & Czech & NR & $317 / 613$ & $307 / 627$ & $42 / 136$ & 0.18 & $281 / 583$ & $309 / 638$ & $76 / 156$ & 0.35 & 1 \\
\hline Vossen et al & 2011 & Germany & $\begin{array}{l}\text { Colon } \\
\text { Rectum }\end{array}$ & $\begin{array}{l}454 / 795 \\
283 / 795\end{array}$ & $\begin{array}{l}502 / 807 \\
321 / 807\end{array}$ & $\begin{array}{l}122 / 209 \\
80 / 209\end{array}$ & 0.85 & & & & & 39 \\
\hline Lee et al & 2012 & USA/NHS & NR & $89 / 165$ & $66 / 140$ & $20 / 48$ & 0.04 & & & $21 / 38$ & 0.11 & 41 \\
\hline Lee et al & 2012 & USA/HPFS & NR & $72 / 140$ & $69 / 127$ & $17 / 51$ & 0.02 & $73 / 147$ & $73 / 133$ & $7 / 32$ & 0.81 & 41 \\
\hline Lee et al & 2012 & USA/PHS & NR & $89 / 159$ & $94 / 124$ & $15 / 50$ & 0.00 & $101 / 167$ & $100 / 154$ & $12 / 44$ & 0.36 & 41 \\
\hline
\end{tabular}

Asia

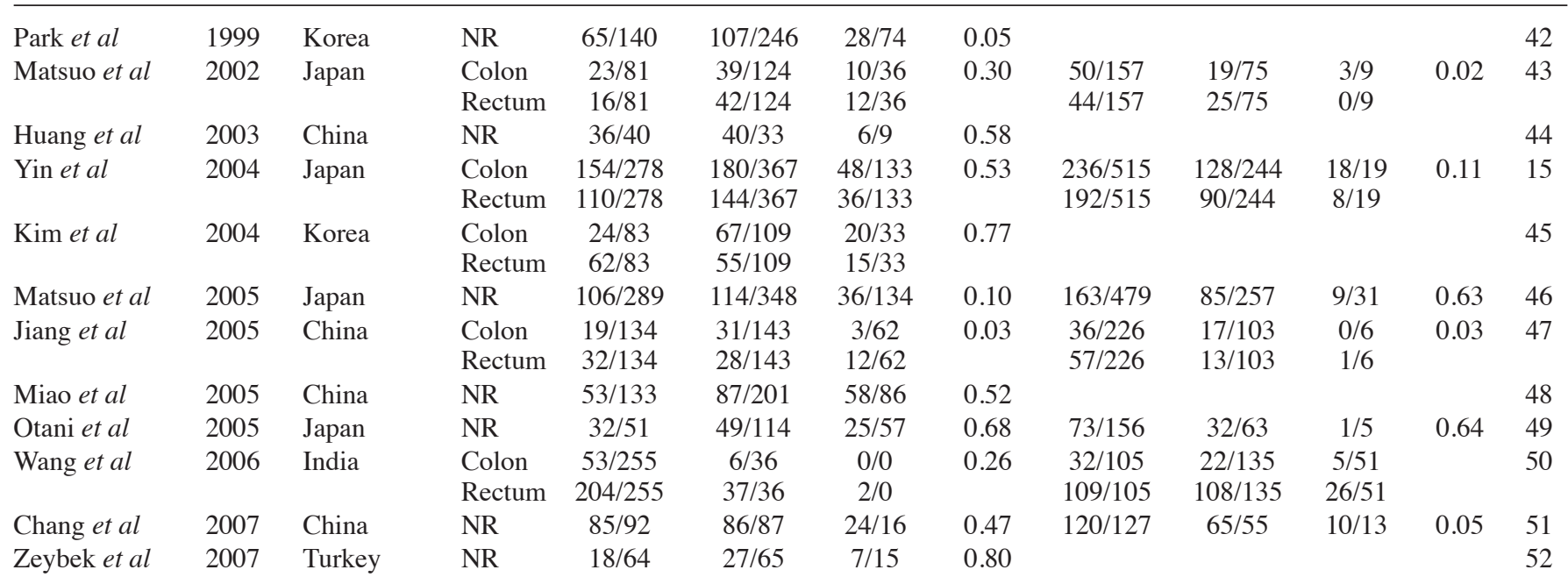


Table I. Continued.

MTHFR 677C/T (case/control) MTHFR 1298A/C (case/control)

\begin{tabular}{llllllllllllll}
\cline { 3 - 4 } & Yearst author & Country & Location & CC & CT & TT & P-value & AA & AC & CC & P-value Refs
\end{tabular}

\begin{tabular}{|c|c|c|c|c|c|c|c|c|c|c|c|c|}
\hline \multicolumn{13}{|l|}{ Asia } \\
\hline Jin et al & 2007 & China & $\begin{array}{l}\text { Colon } \\
\text { Rectum }\end{array}$ & $\begin{array}{l}99 / 211 \\
82 / 211\end{array}$ & $\begin{array}{c}117 / 325 \\
92 / 325\end{array}$ & $\begin{array}{l}35 / 136 \\
20 / 136\end{array}$ & 0.60 & & & & & 53 \\
\hline Cao et al & 2008 & China & $\begin{array}{l}\text { Colon } \\
\text { Rectum }\end{array}$ & $\begin{array}{l}30 / 121 \\
79 / 121\end{array}$ & $\begin{array}{c}53 / 183 \\
101 / 183\end{array}$ & $\begin{array}{l}22 / 66 \\
30 / 66\end{array}$ & 0.82 & $\begin{array}{c}66 / 239 \\
138 / 239\end{array}$ & $\begin{array}{l}38 / 119 \\
67 / 119\end{array}$ & $\begin{array}{l}1 / 13 \\
5 / 13\end{array}$ & 0.70 & 2 \\
\hline Mokarram et al & 2008 & Iran & Colon & $64 / 40$ & $80 / 31$ & $7 / 10$ & 0.31 & & & & & 54 \\
\hline Zhang et al & 2008 & China & NR & $97 / 91$ & $136 / 139$ & $67 / 69$ & 0.26 & & & & & 56 \\
\hline Haghighi et al & 2009 & Iran & NR & $117 / 94$ & $68 / 80$ & $49 / 83$ & 0.00 & & & & & 55 \\
\hline Promthet et al & 2010 & Thailand & Colon & $104 / 94$ & $26 / 31$ & $0 / 5$ & 0.24 & $43 / 54$ & $84 / 71$ & $3 / 5$ & 0.00 & 57 \\
\hline Cui et al & 2010 & Korea & NR & $622 / 540$ & $923 / 863$ & $284 / 297$ & 0.13 & & & & & 6 \\
\hline $\begin{array}{l}\text { Naghibalhos- } \\
\text { saini et al }\end{array}$ & 2010 & Iran & Colon & $64 / 150$ & $80 / 68$ & $7 / 13$ & 0.16 & & & & & 58 \\
\hline Chandy et al & 2010 & India & NR & $74 / 66$ & $25 / 19$ & $1 / 1$ & 0.78 & $22 / 22$ & $70 / 50$ & $8 / 14$ & 0.11 & 60 \\
\hline Zhu et al & 2010 & China & NR & $88 / 50$ & $102 / 53$ & $26 / 8$ & 0.23 & & & & & 61 \\
\hline Yang et al & 2010 & China & NR & $58 / 62$ & $61 / 75$ & $22 / 28$ & 0.52 & & & & & 75 \\
\hline Kim et al & 2011 & Korea & NR & $30 / 15$ & $30 / 21$ & $7 / 17$ & 0.13 & $44 / 36$ & $22 / 16$ & $1 / 1$ & 0.61 & 59 \\
\hline Zhu et al & 2011 & China & NR & $29 / 49$ & $42 / 41$ & $15 / 10$ & 0.74 & & & & & 76 \\
\hline $\begin{array}{l}\text { Kang et al } \\
\text { Prasad and }\end{array}$ & 2011 & Korea & NR & $87 / 145$ & $134 / 238$ & $34 / 65$ & 0.04 & & & & & 62 \\
\hline Wilkhoo & 2011 & India & NR & $97 / 228$ & $12 / 12$ & $1 / 1$ & 0.07 & & & & & 63 \\
\hline Sameer et al & 2011 & India & $\begin{array}{l}\text { Colon } \\
\text { Rectum }\end{array}$ & $\begin{array}{l}23 / 121 \\
36 / 121\end{array}$ & $\begin{array}{c}7 / 27 \\
11 / 27\end{array}$ & $\begin{array}{l}6 / 12 \\
3 / 12\end{array}$ & 0.00 & & & & & 64 \\
\hline Li et al & 2011 & China & NR & $68 / 55$ & $54 / 64$ & $15 / 26$ & 0.33 & $88 / 76$ & $47 / 60$ & $2 / 9$ & 0.53 & 65 \\
\hline Yin et al & 2012 & China & NR & $124 / 139$ & $167 / 178$ & $79 / 53$ & 0.74 & & & & & 66 \\
\hline Kim et al & 2012 & Korea & $\begin{array}{l}\text { Colon } \\
\text { Rectum }\end{array}$ & $\begin{array}{l}121 / 205 \\
109 / 205\end{array}$ & $\begin{array}{l}185 / 289 \\
164 / 289\end{array}$ & $\begin{array}{l}57 / 162 \\
57 / 162\end{array}$ & 0.00 & & & & & 67 \\
\hline
\end{tabular}

Africa

\begin{tabular}{|c|c|c|c|c|c|c|c|c|c|c|c|c|}
\hline Keku et al & 2002 & USA & NR & $198 / 264$ & $43 / 59$ & $3 / 6$ & 0.21 & $157 / 217$ & 78/99 & $8 / 13$ & 0.69 & 12 \\
\hline Lima et al & 2007 & Brazil & NR & 4/143 & $5 / 127$ & $1 / 30$ & 0.82 & $5 / 191$ & $4 / 93$ & $1 / 16$ & 0.30 & 28 \\
\hline El Awady et al & 2009 & Egypt & NR & $6 / 44$ & $23 / 20$ & $6 / 4$ & 0.41 & $5 / 26$ & $21 / 37$ & $9 / 15$ & 0.09 & 68 \\
\hline Guimarães et al & 2011 & Brazil & $\begin{array}{l}\text { Colon } \\
\text { Rectum }\end{array}$ & $\begin{array}{l}17 / 92 \\
31 / 92\end{array}$ & $\begin{array}{l}28 / 79 \\
22 / 79\end{array}$ & $\begin{array}{l}9 / 17 \\
6 / 17\end{array}$ & 0.99 & $\begin{array}{l}39 / 127 \\
28 / 127\end{array}$ & $\begin{array}{l}12 / 49 \\
26 / 49\end{array}$ & $\begin{array}{l}3 / 12 \\
5 / 12\end{array}$ & 0.02 & 69 \\
\hline
\end{tabular}

Mixed

\begin{tabular}{|c|c|c|c|c|c|c|c|c|c|c|c|c|}
\hline Slattery et al & 1999 & USA & Colon & $673 / 827$ & $655 / 787$ & $139 / 207$ & 0.34 & & & & & 70 \\
\hline Curtin et al & 2004 & USA & Colon & $734 / 887$ & $724 / 858$ & $150 / 227$ & 0.37 & $757 / 929$ & $698 / 827$ & $153 / 216$ & 0.12 & 71 \\
\hline $\begin{array}{l}\text { Le Marchand } \\
\text { et al }\end{array}$ & 2005 & USA & $\begin{array}{l}\text { Colon } \\
\text { Rectum }\end{array}$ & $\begin{array}{c}295 / 987 \\
99 / 987\end{array}$ & $\begin{array}{c}246 / 779 \\
90 / 779\end{array}$ & $\begin{array}{l}56 / 255 \\
31 / 255\end{array}$ & 0.00 & & & & & 77 \\
\hline Murtaugh et al & 2007 & USA & Rectum & $357 / 466$ & $301 / 392$ & $84 / 112$ & 0.04 & $360 / 436$ & $317 / 424$ & $65 / 110$ & 0.65 & 72 \\
\hline Iacopetta et al & 2009 & Australia & NR & $382 / 428$ & $386 / 429$ & $82 / 101$ & 0.67 & & & & & 73 \\
\hline Reeves et al & 2009 & Australia & NR & $105 / 101$ & $83 / 91$ & $18 / 19$ & 0.82 & $92 / 86$ & $89 / 98$ & $25 / 27$ & 0.91 & 74 \\
\hline
\end{tabular}

NR, not reported; P-value is for Hardy-Weinberg equilibrium.

95\% CI: 0.76-0.96; TT vs.CC+CT: OR=0.85; 95\% CI: 0.76-0.95) (1298CC vs. AA: OR=0.82; 95\% CI: 0.69-0.98; and $\mathrm{CC}$ vs. $\mathrm{AA}+\mathrm{AC}: \mathrm{OR}=0.83 ; 95 \% \mathrm{CI}: 0.70-0.99)$.

Furthermore, the participants were stratified by tumor location. A significantly decreased risk of CRC was observed under the recessive model of MTHFR 677TT $(\mathrm{OR}=0.83$; 95\% CI: 0.72-0.96) and 1298CC (OR=0.81; 95\% CI: 0.69-0.95) in the colon cancer group. In addition, the stratified analysis revealed that MTHFR C677T was associated with reduced risk of rectal cancer. The OR under the recessive model was 0.86 (95\% CI: 0.77-0.97). The main results are presented in Table II.

Sensitivity analysis and publication bias. The elimination of each individual study imparted no qualitative difference on the pooled OR values, indicating that the final results of the meta-analysis were relatively stable. The publication bias of the studies was determined by a funnel plot and the Egger's 


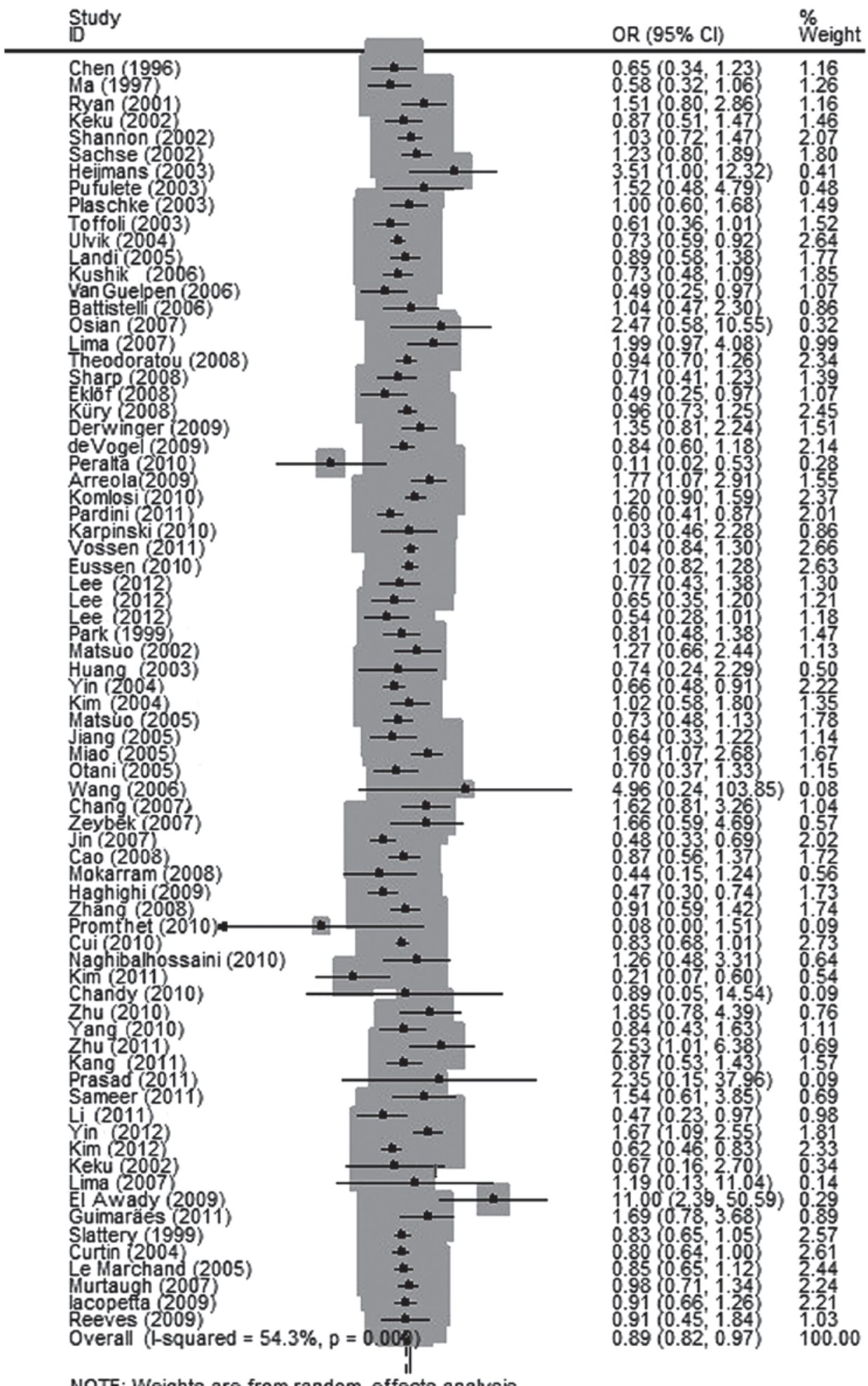

NOTE: Weights are from random-effects analysis

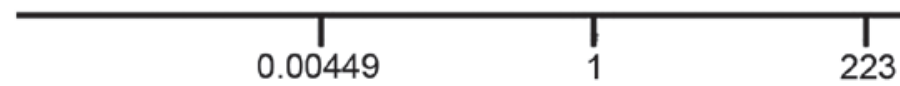

Figure 1. Forest plot for the association between methylenetetrahydrofolate reductase C677T polymorphism and colorectal cancer risk for the TT genotype compared to the $\mathrm{CC}$ genotype in the overall population. OR, odds ratio; CI, confidence interval.

test. The shapes of the funnel plot for each comparison indicated no obvious asymmetry (Figs. 3 and 4) and the Egger's test was then used to provide statistical evidence for the funnel plot symmetry. No significant publication bias was detected in the studies. The results are presented in Table III.

\section{Discussion}

The first study to evaluate the association between the MTHFR C677T polymorphism and CRC was conducted by Chen et al (10). The findings of that study suggested that the 


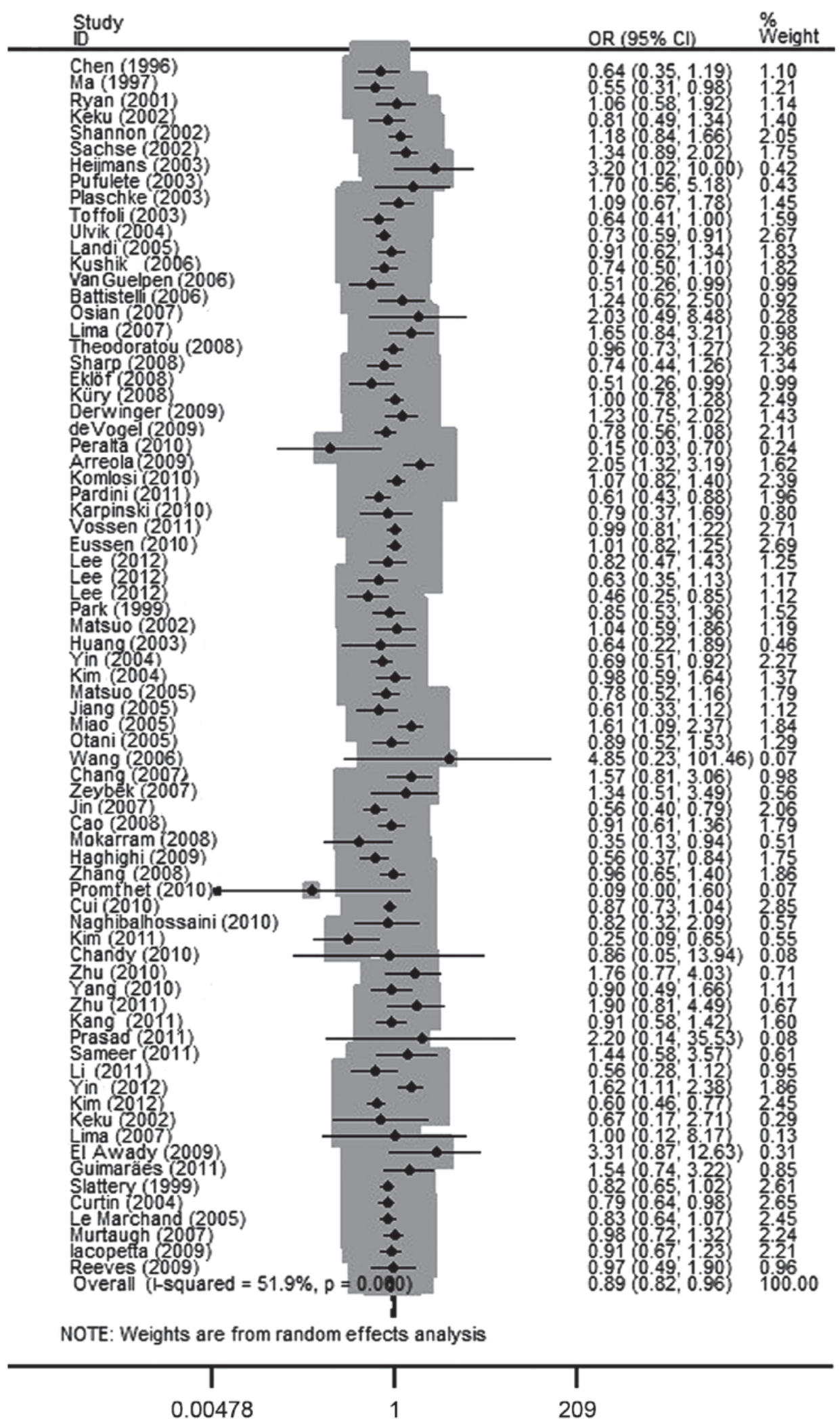

Figure 2. Forest plot for the association between methylenetetrahydrofolate reductase C677T polymorphism and colorectal cancer risk for the TT genotype compared to the $\mathrm{CC}+\mathrm{CT}$ genotype in the overall population. $\mathrm{OR}$, odds ratio; $\mathrm{CI}$, confidence interval.

MTHFR C677T mutation affected enzyme activity and was involved in abnormal methylation as well as DNA synthesis, leading to colorectal tumorigenesis. Similar results were subsequently reported by Ma et al (16), Slattery et al (70) and Le Marchand et al (77). In addition, Le Marchand et al (77) observed that the MTHFR $1298 \mathrm{C}$ allele was weakly protective against CRC. However, Guimarães et al (69) reported that the carriers of the combined variants MTHFR $1298 \mathrm{AC}+\mathrm{CC}$ and 677CT+TT exhibited an increased risk of CRC, whether in isolation or in combination. According to Shannon et al (14) 
Table II. Association of MTHFR C677T and A1298C polymorphisms with CRC risk.

\begin{tabular}{|c|c|c|c|c|c|c|c|c|}
\hline \multirow[b]{2}{*}{ C677T } & \multicolumn{2}{|c|}{ TT vs. CC } & \multicolumn{2}{|c|}{ CT vs. CC } & \multicolumn{2}{|c|}{ TT vs. $\mathrm{CC}+\mathrm{CT}$} & \multicolumn{2}{|c|}{ TT+CT vs. CC } \\
\hline & OR $(95 \% \mathrm{CI})$ & P-value & OR $(95 \% \mathrm{CI})$ & P-value & OR $(95 \% \mathrm{CI})$ & P-value & OR $(95 \% \mathrm{CI})$ & P-value \\
\hline Total & $0.89(0.45-0.97)$ & 0.009 & $1.02(0.97-1.07)$ & 0.378 & $0.89(0.82-0.96)$ & 0.003 & $1.00(0.95-1.05)$ & 0.889 \\
\hline \multicolumn{9}{|l|}{ Ethnicity } \\
\hline European & $0.90(0.80-1.02)$ & 0.088 & $1.02(0.95-1.09)$ & 0.661 & $0.90(0.80-1.01)$ & 0.075 & $0.99(0.93-1.06)$ & 0.831 \\
\hline Asian & $0.86(0.73-1.02)$ & 0.087 & $1.03(0.93-1.13)$ & 0.583 & $0.87(0.75-1.01)$ & 0.059 & $1.00(0.90-1.11)$ & 0.981 \\
\hline African & $1.96(0.66-5.85)$ & 0.225 & $1.75(0.81-3.78)$ & 0.153 & $1.49(0.85-2.62)$ & 0.165 & $1.80(0.82 .3 .94)$ & 0.143 \\
\hline Mixed & $0.86(0.76-0.96)$ & 0.010 & $1.02(0.95-1.10)$ & 0.557 & $0.85(0.76-0.95)$ & 0.004 & $0.99(0.92-1.06)$ & 0.703 \\
\hline \multicolumn{9}{|c|}{ Tumor location } \\
\hline Colon & $0.86(0.73-1.02)$ & 0.079 & $1.08(0.97-1.20)$ & 0.161 & $0.83(0.72-0.96)$ & 0.014 & $1.03(0.93-1.15)$ & 0.566 \\
\hline \multirow[t]{2}{*}{ Rectum } & $0.88(0.73-1.07)$ & 0.208 & $1.07(0.98-1.16)$ & 0.129 & $0.86(0.77-0.97)$ & 0.014 & $1.01(0.89-1.15)$ & 0.880 \\
\hline & \multicolumn{2}{|c|}{ CC vs. AA } & \multicolumn{2}{|c|}{ AC vs. AA } & \multicolumn{2}{|c|}{$\mathrm{CC}$ vs. $\mathrm{AA}+\mathrm{AC}$} & \multicolumn{2}{|c|}{$\mathrm{CC}+\mathrm{AC}$ vs. AA } \\
\hline A1298C & OR $(95 \% \mathrm{CI})$ & P-value & OR $(95 \% \mathrm{CI})$ & P-value & OR $(95 \% \mathrm{CI})$ & P-value & OR $(95 \% \mathrm{CI})$ & P-value \\
\hline Total & $0.92(0.81-1.05)$ & 0.202 & $1.03(0.98-1.08)$ & 0.221 & $0.90(0.80-1.01)$ & 0.086 & $1.02(0.96-1.08)$ & 0.619 \\
\hline \multicolumn{9}{|l|}{ Ethnicity } \\
\hline European & $1.01(0.91-1.11)$ & 0.864 & $1.04(0.98-1.11)$ & 0.169 & $0.99(0.90-1.09)$ & 0.841 & $1.04(0.98-1.10)$ & 0.208 \\
\hline Asian & $0.69(0.54-0.89)$ & 0.004 & $1.00(0.89-1.11)$ & 0.984 & $0.69(0.54-0.88)$ & 0.002 & $0.96(0.86-1.07)$ & 0.466 \\
\hline African & $1.92(0.70-5.31)$ & 0.207 & $1.33(0.96-1.83)$ & 0.082 & $1.50(0.70-3.20)$ & 0.294 & $1.45(0.94-2.23)$ & 0.091 \\
\hline Mixed & $0.82(0.69-0.98)$ & 0.033 & $0.98(0.88-1.10)$ & 0.730 & $0.83(0.70-0.99)$ & 0.035 & $0.95(0.85-1.05)$ & 0.316 \\
\hline \multicolumn{9}{|c|}{ Tumor location } \\
\hline Colon & $0.81(0.59-1.12)$ & 0.200 & $1.03(0.94-1.13)$ & 0.535 & $0.81(0.69-0.95)$ & 0.012 & $0.99(0.91-1.08)$ & 0.872 \\
\hline Rectum & $0.85(0.50-1.42)$ & 0.530 & $1.00(0.79-1.26)$ & 0.980 & $0.85(0.55-1.32)$ & 0.474 & $0.95(0.74-1.23)$ & 0.719 \\
\hline
\end{tabular}

CRC, colorectal cancer; OR, odds ratio; CI, confidence interval.

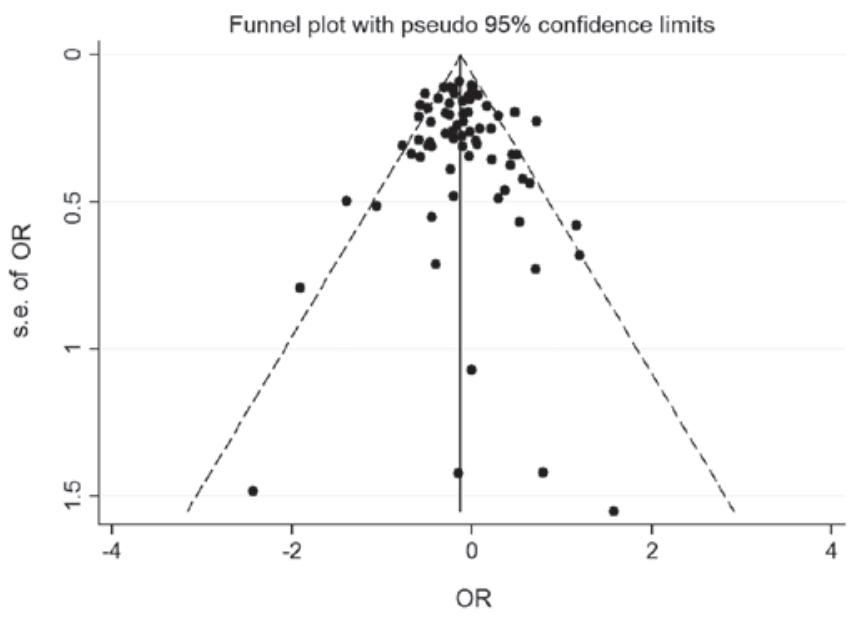

Figure 3. Funnel plot with pseudo 95\% confidence limits on the association between methylenetetrahydrofolate reductase $\mathrm{C} 677 \mathrm{~T}$ polymorphism and colorectal cancer risk. SE, standard error; OR, odds ratio.

and Prasad et al (63), the MTHFR polymorphism C677T was a risk factor for CRC development. Furthermore, several other published studies failed to support an effect of MTHFR gene polymorphisms on CRC risk $(43,49,60)$, due to statistically non-significant results.

The conflicting conclusions among the studies mentioned above may be attributed to several causes. First, the sample sizes of the populations included in several studies were

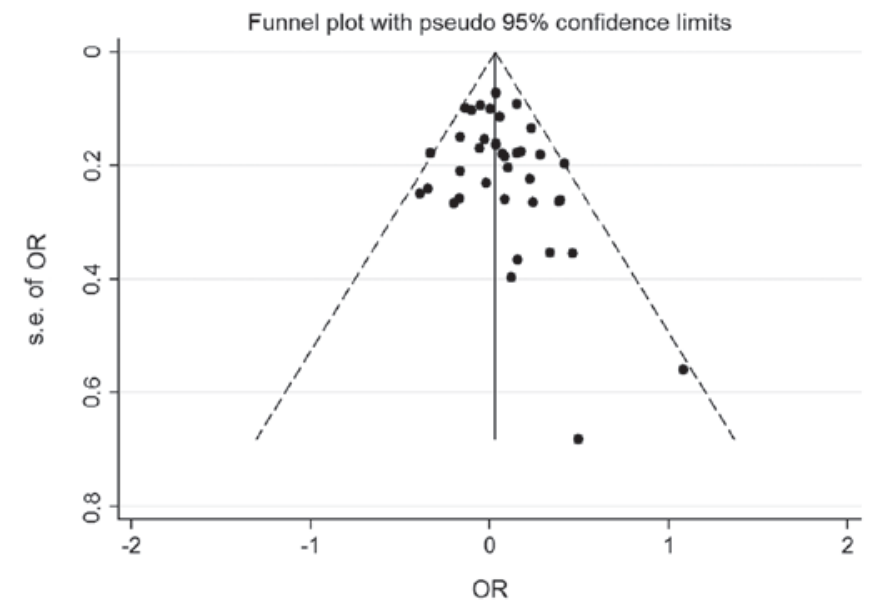

Figure 4. Funnel plot with pseudo $95 \%$ confidence limits on the association between methylenetetrahydrofolate reductase A1298C polymorphism and colorectal cancer risk. SE, standard error; OR, odds ratio.

relatively small $(19,27,69)$, which may result in false-positive or false-negative outcomes. Second, the eligibility criteria for inclusion of control subjects differed among the studies. Certain studies were hospital-based $(35,45,50)$, whereas others were population-based $(12,13,47,63)$. Therefore, some controls were non-cancer cases, whereas others were healthy individuals. The inclusion of individuals from different ethnic backgrounds should also be considered. 
Table III. Results of Egger's test for the models.

\begin{tabular}{|c|c|c|c|c|c|}
\hline Polymorphism & Model & T-value & P-value & \multicolumn{2}{|c|}{$95 \%$ CI of intercept value } \\
\hline \multicolumn{6}{|l|}{ MTHFR C677T } \\
\hline & TT vs. CC & 1.04 & 0.303 & -0.3457011 & 1.096597 \\
\hline & CT vs. CC & 1.86 & 0.067 & -0.0274857 & 0.7770219 \\
\hline & TT vs. $\mathrm{CC}+\mathrm{CT}$ & 0.62 & 0.535 & -0.485652 & 0.9266581 \\
\hline & $\mathrm{TT}+\mathrm{CT}$ vs. $\mathrm{CC}$ & 1.66 & 0.102 & -0.1176829 & 1.27792 \\
\hline \multicolumn{6}{|c|}{ MTHFR A1298C } \\
\hline & CC vs. AA & -0.52 & 0.605 & -0.9875751 & 0.5834467 \\
\hline & AC vs. AA & 1.57 & 0.125 & -0.1645624 & 1.29779 \\
\hline & $\mathrm{CC}$ vs. $\mathrm{AA}+\mathrm{AC}$ & -1.02 & 0.312 & -1.094435 & 0.3594053 \\
\hline & $\mathrm{CC}+\mathrm{AC}$ vs. AA & 1.13 & 0.266 & -0.3669942 & 1.292081 \\
\hline
\end{tabular}

CI, confidence interval.

As regards the conflicting results, we performed a meta-analysis to elucidate the association of MTHFR C677T and A1298C polymorphisms with CRC risk and to provide a comprehensive assessment. In this meta-analysis, the pooled results indicated that the homozygous variant of MTHFR C677T polymorphism exerted a protective effect against $\mathrm{CRC}$ development $(\mathrm{OR}=0.89$; 95\% CI: 0.82-0.97). However, when analysis was performed by ethnicity, this effect was not observed in all the subgroups, except for the mixed population (OR=0.86; 95\% CI: 0.76-0.96). Additionally, a significant association was observed between MTHFR $1298 \mathrm{CC}$ and $\mathrm{CRC}$ in the mixed population (OR=0.82; 95\% CI: 0.69-0.98). When this analysis was restricted by limiting studies to Asian populations, the MTHFR 1298CC genotype exhibited a decreased risk of CRC, with an OR of 0.69 (95\% CI: 0.54-0.89). In the subanalysis by tumor location it was demonstrated that individuals with the MTHFR 677TT genotype exhibited a decreased risk of colon and rectal cancer, with an OR of 0.83 (95\% CI: 0.72-0.96) and 0.86 (95\% CI: 0.77-0.97), respectively. It was also confirmed that the MTHFR A1298C polymorphism is involved in colon cancer development ( $\mathrm{CC}$ vs. $\mathrm{AA}+\mathrm{AC}$ : $\mathrm{OR}=0.81 ; 95 \% \mathrm{CI}$ : 0.69-0.96). Our findings in this meta-analysis were consistent with the results reported by the majority of the published studies.

According to Begg's funnel plots and Egger's test, there was no significant publication bias in the present meta-analysis. However, there was obvious heterogeneity, which was a potential problem when interpreting the results of the meta-analysis. Several sources of heterogeneity should be considered. First, different selection criteria of cases and controls, as well as gender and age distribution, may affect between-study heterogeneity. Second, there was some diversity among the studies regarding design, sample size and family history. Ethnic variations were also crucial as it was demonstrated that the heterogeneity was decreased when analyzed by ethnicity in this meta-analysis. The different ethnicities were distinguished according to geography; however, potential confounding factors, such as genetic background, lifestyle and dietary habits, could not be excluded.

The present study has certain limitations. First, the criteria for inclusion of controls differed among the studies. The controls in certain studies were selected from healthy indi- viduals, whereas the controls in other studies were selected from non-cancer cases. Second, our results were based on unadjusted OR values, which may lead to relatively low power of the estimation of the real association. The gender and age distribution of the participants, the dietary pattern, the folate status, alcohol consumption and other risk factors may affect between-study heterogeneity. An analysis should be conducted to obtain adjusted ORs for other covariates, such as age, gender and folate status, provided more individual study data are available. Third, the subgroup analyses had insufficient statistical power to detect the association. The gene-gene and gene-environment interactions may affect the association between MTHFR polymorphisms and CRC. According to the study by Keku et al (12), the combination of MTHFR 677CC and 1298AA genotypes exhibited an increased risk of CRC. In addition, Ma et al (16) and Kim et al (67) demonstrated that a high folate intake was associated with reduced risk of CRC and high alcohol consumption was associated with an increased risk of CRC. The gene-gene and gene-environment interactions could not be examined due to unavailability of individual data.

Despite the limitations described above, our meta-analysis also has certain advantages. The substantial number of cases and controls were pooled from different studies, which provided more reliable evidence on the association between MTHFR polymorphisms and the risk of CRC. In addition, the study subjects were classified into colon and rectal cancer groups, in order to exclude certain confounding factors. The pooled data clearly demonstrated that MTHFR C677T significantly affected carcinogenesis in the colon and rectum, whereas A1298C appeared to be mainly associated with rectal tumorigenesis. This finding may be attributed to different carcinogenic mechanisms underlying colon and rectal cancer.

In conclusion, the results of this meta-analysis indicated a significant association of the MTHFR C677T and A1298C polymorphisms with the risk of CRC. Particularly, the MTHFR 677T and $1298 \mathrm{C}$ alleles were associated with a low risk of CRC.

\section{Acknowledgements}

This study was supported by grants no. 81102194 and no. 81272293 from the National Natural Science Foundation 
of China, grant no. LS2010168 from the Liaoning Provincial Department of Education and grant no. 00726 from the China Medical Board. The authors are grateful to all the participants in this study.

\section{References}

1. Pardini B, Kumar R, Naccarati A, Prasad RB, Forsti A, Polakova V, Vodickova L, Novotny J, Hemminki K and Vodicka P: MTHFR and MTRR genotype and haplotype analysis and colorectal cancer susceptibility in a case-control study from the Czech Republic. Mutat Res 721: 74-80, 2011.

2. Cao HX, Gao CM, Takezaki T, Wu JZ, Ding JH, Liu YT, Li SP, Su P, Cao J, Hamajima N and Tajima K: Genetic polymorphisms of methylenetetrahydrofolate reductase and susceptibility to colorectal cancer. Asian Pac J Cancer Prev 9: 203-208, 2008.

3. Jemal A, Bray F, Center MM, Ferlay J, Ward E and Forman D: Global cancer statistics. CA Cancer J Clin 61: 69-90, 2011.

4. Blount BC, Mack MM, Wehr CM, MacGregor JT, Hiatt RA, Wang G, Wickramasinghe SN, Everson RB and Ames BN: Folate deficiency causes uracil misincorporation into human DNA and chromosome breakage: implications for cancer and neuronal damage. Proc Natl Acad Sci USA 94: 3290-3295, 1997.

5. Choi SW and Mason JB: Folate and carcinogenesis: an integrated scheme. J Nutr 130: 129-132, 2000.

6. CuiLH, Shin MH, Kweon SS, KimHN, Song HR, Piao JM, Choi JS, Shim HJ, Hwang JE, Kim HR, et al: Methylenetetrahydrofolate reductase $\mathrm{C} 677 \mathrm{~T}$ polymorphism in patients with gastric and colorectal cancer in a Korean population. BMC Cancer 10: 236, 2010.

7. Goyette P, Sumner JS, Milos R, Duncan AM, Rosenblatt DS, Matthews RG and Rozen R: Human methylenetetrahydrofolate reductase: isolation of cDNA, mapping and mutation identification. Nat Genet 7: 195-200, 1994.

8. Pereira AC, Schettert IT, Morandini Filho AA, GuerraShinohara EM and Krieger JE: Methylenetetrahydrofolate reductase (MTHFR) c677t gene variant modulates the homocysteine folate correlation in a mild folate-deficient population. Clin Chim Acta 340: 99-105, 2004.

9. Ogino S and Wilson RB: Genotype and haplotype distributions of MTHFR677C $>\mathrm{T}$ and 1298A $>\mathrm{C}$ single nucleotide polymorphisms: a meta-analysis. J Hum Genet 48: 1-7, 2003.

10. Chen J, Giovannucci E, Kelsey K, et al: A methylenetetrahydrofolate reductase polymorphism and the risk of colorectal cancer. Cancer Res 56: 4862-4864, 1996.

11. van der Put NM, Gabreels F, Stevens EM, Smeitink JA, Trijbels FJ, Eskes TK, van den Heuvel LP and Blom HJ: A second common mutation in the methylenetetrahydrofolate reductase gene: an additional risk factor for neural-tube defects? Am J Hum Genet 62: 1044-1051, 1998.

12. Keku T, Millikan R, Worley K, Winkel S, Eaton A, Biscocho L, Martin C and Sandler R: 5,10-Methylenetetrahydrofolate reductase codon 677 and 1298 polymorphisms and colon cancer in African Americans and whites. Cancer Epidemiol Biomarkers Prev 11: 1611-1621, 2002.

13. Heijmans BT, Boer JM, Suchiman HE, Cornelisse CJ, Westendorp RG, Kromhout D, Feskens EJ and Slagboom PE: A common variant of the methylenetetrahydrofolate reductase gene (1p36) is associated with an increased risk of cancer. Cancer Res 63: 1249-1253, 2003.

14. Shannon B, Gnanasampanthan S, Beilby J and Iacopetta B: A polymorphism in the methylenetetrahydrofolate reductase gene predisposes to colorectal cancers with microsatellite instability. Gut 50: 520-524, 2002.

15. Yin $\mathrm{G}$, Kono $\mathrm{S}$, Toyomura $\mathrm{K}$, Hagiwara $\mathrm{T}$, Nagano $\mathrm{J}$, Mizoue T, Mibu R, Tanaka M, Kakeji Y, Maehara Y, et al: Methylenetetrahydrofolate reductase C677T and A1298C polymorphisms and colorectal cancer: the Fukuoka Colorectal Cancer Study. Cancer Sci 95: 908-913, 2004.

16. Ma J, Stampfer MJ, Giovannucci E, et al: Methylenetetrahydrofolate reductase polymorphism, dietary interactions, and risk of colorectal cancer. Cancer Res 57: 1098-1102, 1997.

17. Ryan BM, Molloy AM, McManus R, Arfin Q, Kelleher D, Scott JM and Weir DG: The methylenetetrahydrofolate reductase (MTHFR) gene in colorectal cancer: role in tumor development and significance of allelic loss in tumor progression. Int $\mathrm{J}$ Gastrointest Cancer 30: 105-111, 2001.
18. Sachse C, Smith G, Wilkie MJ, Barrett JH, Waxman R, Sullivan F, Forman D, Bishop DT and Wolf CR; Colorectal Cancer Study Group: A pharmacogenetic study to investigate the role of dietary carcinogens in the etiology of colorectal cancer. Carcinogenesis 23: 1839-1849, 2002.

19. Pufulete M, Al-Ghnaniem R, Leather AJ, Appleby P, Gout S, Terry C, Emery PW and Sanders TA: Folate status, genomic DNA hypomethylation, and risk of colorectal adenoma and cancer: a case control study. Gastroenterology 124: 1240-1248, 2003.

20. Plaschke J, Schwanebeck U, Pistorius S, Saeger HD and Schackert HK: Methylenetetrahydrofolate reductase polymorphisms and risk of sporadic and hereditary colorectal cancer with or without microsatellite instability. Cancer Lett 191: 179-185, 2003.

21. Toffoli G, Gafà R, Russo A, Lanza G, Dolcetti R, Sartor F, Libra M, Viel A and Boiocchi M: Methylenetetrahydrofolate reductase $677 \mathrm{C} \rightarrow \mathrm{T}$ polymorphism and risk of proximal colon cancer in north Italy. Clin Cancer Res 9: 743-748, 2003.

22. Ulvik A, Vollset SE, Hansen S, Gislefoss R, Jellum E and Ueland PM: Colorectal cancer and the methylenetetrahydrofolate reductase $677 \mathrm{C} \rightarrow \mathrm{T}$ and methionine synthase $2756 \mathrm{~A} \rightarrow \mathrm{G}$ polymorphisms: a study of 2,168 case-control pairs from the JANUS cohort. Cancer Epidemiol Biomarkers Prev 13: 2175-2180, 2004.

23. Landi S, Gemignani F, Moreno V, Gioia-Patricola L, Chabrier A, Guino E, Navarro M, de Oca J, Capellà G and Canzian F; Bellvitge Colorectal Cancer Study Group: A comprehensive analysis of phase I and phase II metabolism gene polymorphisms and risk of colorectal cancer. Pharmacogenet Genomics 15: 535-546, 2005.

24. Koushik A, Kraft P, Fuchs CS, Hankinson SE, Willett WC, Giovannucci EL and Hunter DJ: Nonsynonymous polymorphisms in genes in the one-carbon metabolism pathway and associations with colorectal cancer. Cancer Epidemiol Biomarkers Prev 15: 2408-2017, 2006

25. Van Guelpen B, Hultdin J, Johansson I, Hallmans G, Stenling R, Riboli E, Winkvist A and Palmqvist R: Low folate levels may protect against colorectal cancer. Gut 55: 1461-1466, 2006.

26. Battistelli S, Vittoria A, Stefanoni M, Bing C and Roviello F: Total plasma homocysteine and methylenetetrahydrofolate reductase $\mathrm{C} 677 \mathrm{~T}$ polymorphism in patients with colorectal carcinoma. World J Gastroenterol 12: 6128-6132, 2006.

27. Osian G, Procopciuc L and Vlad L: MTHFR polymorphisms as prognostic factors in sporadic colorectal cancer. J Gastrointestin Liver Dis 16: 251-256, 2007.

28. Lima CS, Nascimento H, Bonadia LC, Teori MT, Coy CS, Goes JR, Costa FF and Bertuzzo CS: Polymorphisms in methylenetetrahydrofolate reductase gene (MTHFR) and the age of onset of sporadic colorectal adenocarcinoma. Int $\mathrm{J}$ Colorectal Dis 22: 757-763, 2007.

29. Theodoratou E, Farrington SM, Tenesa A, McNeill G, Cetnarskyj R, Barnetson RA, Porteous ME, Dunlop MG and Campbell H: Dietary vitamin B6 intake and the risk of colorectal cancer. Cancer Epidemiol Biomarkers Prev 17: 171-182, 2008.

30. Sharp L, Little J, Brockton NT, Cotton SC, Masson LF, Haites NE and Cassidy J: Polymorphisms in the methylenetetrahydrofolate reductase (MTHFR) gene, intakes of folate and related B vitamins and colorectal cancer: a case-control study in a population with relatively low folate intake. Br J Nutr 99: 379-389, 2008

31. Eklöf V, Van Guelpen B, Hultdin J, Johansson I, Hallmans G and Palmqvist R: The reduced folate carrier (RFC1) 80G > A and folate hydrolase 1 (FOLH1) $1561 \mathrm{C}>\mathrm{T}$ polymorphisms and the risk of colorectal cancer: a nested case-referent study. Scand J Clin Lab Invest 68: 393-401, 2008.

32. Küry S, Buecher B, Robiou-du-Pont S, Scoul C, Colman H, Le Neel T, Le Houérou C, Faroux R, Ollivry J, Lafraise B, Chupin LD, Sébille V and Bézieau S: Low-penetrance alleles predisposing to sporadic colorectal cancers: a French case-controlled genetic association study. BMC Cancer 8: 326, 2008.

33. Derwinger K, Wettergren Y, Odin E, Carlsson G and Gustavsson B: A study of the MTHFR gene polymorphism C677T in colorectal cancer. Clin Colorectal Cancer 8: 43-48, 2009.

34. de Vogel S, Wouters KA, Gottschalk RW, van Schooten FJ, de Goeij AF, de Bruïne AP, Goldbohm RA, van den Brandt PA, Weijenberg MP and van Engeland M: Genetic variants of methyl metabolizing enzymes and epigenetic regulators: associations with promoter $\mathrm{CpG}$ island hypermethylation in colorectal cancer. Cancer Epidemiol Biomarkers Prev 18: 3086-3096, 2009. 
35. Fernández-Peralta AM, Daimiel L, Nejda N, Iglesias D, Medina Arana V and González-Aguilera JJ: Association of polymorphisms MTHFR C677T and A1298C with risk of colorectal cancer, genetic and epigenetic characteristic of tumors, and response to chemotherapy. Int J Colorectal Dis 25 : $141-151,2010$.

36. Gallegos-Arreola MP, Garcia-Ortiz JE, Figuera LE, Puebla-Perez AM, Morgan-Villela G and Zuniga-Gonzalez GM: Association of the $677 \mathrm{C} \rightarrow$ T polymorphism in the MTHFR gene with colorectal cancer in Mexican patients. Cancer Genomics Proteomics 6: 183-188, 2009.

37. Komlosi V, Hitre E, Pap E, Adleff V, Reti A, Szekely E, Biro A, Rudnai P, Schoket B, Muller J, et al: SHMT1 1420 and MTHFR 677 variants are associated with rectal but not colon cancer. BMC Cancer 10: 525, 2010.

38. Karpinski P, Myszka A, Ramsey D, Misiak B, Gil J,Laczmanska I, Grzebieniak Z, Sebzda T, Smigiel R, Stembalska A and Sasiadek MM: Polymorphisms in methyl-group metabolism genes and risk of sporadic colorectal cancer with relation to the $\mathrm{CpG}$ island methylator phenotype. Cancer Epidemiol 34: 338-344, 2010

39. Vossen CY, Hoffmeister M, Chang-Claude JC, Rosendaal FR and Brenner H: Clotting factor gene polymorphisms and colorectal cancer risk. J Clin Oncol 29: 1722-1727, 2011.

40. Eussen SJ, Vollset SE, Igland J, Meyer K, Fredriksen A, et al: Plasma folate, related genetic variants, and colorectal cancer risk in EPIC. Cancer Epidemiol Biomarkers Prev 19: 1328-1340, 2010

41. Lee JE, Wei EK, Fuchs CS, Hunter DJ, Lee IM, Selhub J, et al: Plasma folate, methylenetetrahydrofolate reductase (MTHFR), and colorectal cancer risk in three large nested case-control studies. Cancer Causes Control 23: 537-545, 2012.

42. Park KS, Mok JW and Kim JC: The $677 \mathrm{C}>\mathrm{T}$ mutation in 5,10-methylenetetrahydrofolate reductase and colorectal cancer risk. Genet Test 3: 233-236, 1999.

43. Matsuo K, Hamajima N, Hirai T, Kato T, Inoue M, Takezaki T and Tajima K: Methionine synthase reductase gene A66G polymorphism is associated with risk of colorectal cancer. Asian Pac J Cancer Prev 3: 353-359, 2002.

44. Huang P, Zhou ZY, Ma HT, Liu JY, Zhou YH, Cao J, Ge HY Yu PW and Takezaki T: MTHFR polymorphisms and colorectal cancer susceptibility in Chongqing people. Acta Academiae Medicinae Militaris Tertiae 25: 1710-1713, 2003 (In Chinese).

45. Kim DH, Ahn YO, Lee BH, Tsuji E, Kiyohara C and Kono S: Methylenetetrahydrofolate reductase polymorphism, alcohol intake, and risks of colon and rectal cancers in Korea. Cancer Lett 216: 199-205, 2004.

46. Matsuo K, Ito H, Wakai K, Hirose K, Saito T, Suzuki T, Kato T, Hirai T, Kanemitsu Y, Hamajima H and Tajima K: One-carbon metabolism related gene polymorphisms interact with alcohol drinking to influence the risk of colorectal cancer in Japan. Carcinogenesis 26: 2164-2171, 2005.

47. Jiang Q, Chen K, Ma X, Li Q, Yu W, Shu G and Yao K: Diets, polymorphisms of methylenetetrahydrofolate reductase, and the susceptibility of colon cancer and rectal cancer. Cancer Detect Prev 29: 146-154, 2005

48. Miao XP, Yang S, Tan W, Zhang XM, et al: Association between genetic variations in methylenetetrahydrofolate reductase and risk of colorectal cancer in a Chinese population. Chin J Prev Med 39: 409-411, 2005, (In Chinese).

49. Otani T, Iwasaki M, Hanaoka T, Kobayashi M, Ishihara J, Natsukawa S, Shaura K, Koizumi Y, Kasuga Y, Yoshimura K, Yoshida T and Tsugane S: Folate, vitamin B6, vitamin B12, and vitamin B2 intake, genetic polymorphisms of related enzymes, and risk of colorectal cancer in a hospital-based case-control study in Japan. Nutr Cancer 53: 42-50, 2005.

50. Wang J, Gajalakshmi V, Jiang J, Kuriki K, Suzuki S, Nagaya T, Nakamura S, Akasaka S, Ishikawa $\mathrm{H}$ and Tokudome $\mathrm{S}$ Associations between 5,10-methylenetetrahydrofolate reductase codon 677 and 1298 genetic polymorphisms and environmental factors with reference to susceptibility to colorectal cancer: a case-control study in an Indian population. Int J Cancer 118: 991-997, 2006.

51. Chang SC, Lin PC, Lin JK, Yang SH, Wang HS and Li AF: Role of MTHFR polymorphisms and folate levels in different phenotypes of sporadic colorectal cancers. Int J Colorectal Dis 22: 483-489, 2007.

52. Zeybek U, Yaylim I, Yilmaz H, Agaçhan B, Ergen A, Arikan S, Bayrak S and Isbir T: Methylenetetrahydrofolate reductase C677T polymorphism in patients with gastric and colorectal cancer. Cell Biochem Funct 25: 419-422, 2007.
53. Jin XX, Zhu ZZ, Wang AZ and Jia HR: Association of methylenetetrahydrofolate reductase C677T polymorphism with genetic susceptibility to colorectal cancer. World Chin J Digestol 15: 2754-2757, 2007 (In Chinese).

54. Mokarram P, Naghibalhossaini F, Saberi Firoozi M, Hosseini SV, Izadpanah A, Salahi H, Malek-Hosseini SA, Talei A and Mojallal M: Methylenetetrahydrofolate reductase C677T genotype affects promoter methylation of tumor-specific genes in sporadic colorectal cancer through an interaction with folate/vitamin B12 status. World J Gastroenterol 14: 3662-3671, 2008.

55. Haghighi MM, Radpour R, Mahmoudi T, Mohebbi SR, Vahedi M and Zali MR: Association between MTHFR polymorphism (C677T) with nonfamilial colorectal cancer. Oncol Res 18: 57-63, 2009.

56. Zhang YL, Yuan XY, Zhang Z, Yang H, Zhou YH, Pan YM, Zhou ZY, Liang HJ and Cao J: Relationship of genetic polymorphisms in methylenetetrahydrofolate reductase and alcohol drinking with the risk of colorectal cancer. Chin J Cancer Prev Treat 15: 1298-1301, 2008 (In Chinese).

57. Promthet SS, Pientong C, Ekalaksananan T, Wiangnon S, Poomphakwaen K, Songserm N, Chopjitt P, Moore MA and Tokudome S: Risk factors for colon cancer in Northeastern Thailand: interaction of MTHFR codon 677 and 1298 genotypes with environmental factors. J Epidemiol 20: 329-338, 2010

58. Naghibalhossaini F, Mokarram P, Khalili I, Vasei M, Hosseini SV, Ashktorab H, Rasti M and Abdollahi K: MTHFR C677T and A1298C variant genotypes and the risk of microsatellite instability among Iranian colorectal cancer patients. Cancer Genet Cytogenet 197: 142-151, 2010.

59. Kim JW, Park HM, Choi YK, Chong SY, Oh D and Kim NK: Polymorphisms in genes involved in folate metabolism and plasma DNA methylation in colorectal cancer patients. Oncol Rep 25: 167-172, 2011

60. Chandy S, Sadananda Adiga MN, Ramachandra N, Krishnamoorthy S, Ramaswamy G, Savithri HS and Krishnamoorthy L: Association of methylenetetrahydrofolate reductase gene polymorphisms and colorectal cancer in India. Indian J Med Res 131: 659-664, 2010.

61. Zhu F, Wang YM and QY Z: A case-control study of plasma homocysteine, serum folate, the polymorphism of methylenetetrahydrofolate reductase in colorectal cancer. J Southeast Univ Med Sci Edi 29: 88-92, 2010.

62. Kang BS, Ahn DH, Kim NK and Kim JW: Relationship between metabolic syndrome and MTHFR polymorphism in colorectal cancer. J Korean Soc Coloproctol 27: 78-82, 2011.

63. Prasad VV and Wilkhoo $\mathrm{H}$ : Association of the functional polymorphism C677T in the methylenetetrahydrofolate reductase gene with colorectal, thyroid, breast, ovarian, and cervical cancers. Onkologie 34: 422-426, 2011.

64. Sameer AS, Shah ZA, Nissar S, Mudassar S and Siddiqi MA: Risk of colorectal cancer associated with the methylenetetrahydrofolate reductase (MTHFR) C677T polymorphism in the Kashmiri population. Genet Mol Res 10: 1200-1210, 2011.

65. Li H, Xu WL, Shen HL, Chen QY, Hui LL, Long LL and Zhu XL: Methylenetetrahydrofolate reductase genotypes and haplotypes associated with susceptibility to colorectal cancer in an eastern Chinese Han population. Genet Mol Res 10: 3738-3746, 2011

66. Yin G, Ming H, Zheng X, Xuan Y, Liang J and Jin X: Methylenetetrahydrofolate reductase C677T gene polymorphism and colorectal cancer risk: A case-control study. Oncol Lett 4: 365-369, 2012

67. Kim J, Cho YA, Kim DH, Lee BH, Hwang DY, Jeong J, Lee HJ Matsuo K, Tajima K and Ahn YO: Dietary intake of folate and alcohol, MTHFR C677T polymorphism, and colorectal cancer risk in Korea. Am J Clin Nutr 95: 405-412, 2012.

68. El Awady MK,Karim AM,Hanna LS, El Husseiny LA,ElSahar M, Menem HA and Meguid NA: Methylenetetrahydrofolate reductase gene polymorphisms and the risk of colorectal carcinoma in a sample of Egyptian individuals. Cancer Biomark 5: 233-240, 2009.

69. Guimarães JL, Ayrizono Mde L, Coy CS and Lima CS: Gene polymorphisms involved in folate and methionine metabolism and increased risk of sporadic colorectal adenocarcinoma. Tumour Biol 32: 853-861, 2011.

70. Slattery ML, Potter JD, Samowitz W, Schaffer D and Leppert M: Methylenetetrahydrofolate reductase, diet, and risk of colon cancer. Cancer Epidemiol Biomarkers Prev 8: 513-518, 1999. 
71. Curtin K, Bigler J, Slattery ML, Caan B, Potter JD and Ulrich CM: MTHFR C677T and A1298C polymorphisms: diet, estrogen, and risk of colon cancer. Cancer Epidemiol Biomarkers Prev 13: 285-292, 2004

72. Murtaugh MA, Curtin K, Sweeney C, Wolff RK, Holubkov R, Caan BJ and Slattery ML: Dietary intake of folate and co-factors in folate metabolism, MTHFR polymorphisms, and reduced rectal cancer. Cancer Causes Control 18: 153-163, 2007.

73. Iacopetta B, Heyworth J, Girschik J, Grieu F, Clayforth C and Fritschi L: The MTHFR C677T and DeltaDNMT3B C-149T polymorphisms confer different risks for right- and left-sided colorectal cancer. Int J Cancer 125: 84-90, 2009.

74. Reeves SG, Meldrum C, Groombridge C, Spigelman AD, Suchy J, Kurzawski G, Lubinski J, McElduff P and Scott RJ: MTHFR $677 \mathrm{C}>\mathrm{T}$ and $1298 \mathrm{~A}>\mathrm{C}$ polymorphisms and the age of onset of colorectal cancer in hereditary nonpolyposis colorectal cancer. Eur J Hum Genet 17: 629-635, 2009.
75. Yang XX, Li FX, Yi J, Li X, Sun JZ and Hu NY: An association of methylenetetrahydrofolate reductase reductase C677T and gastric cancer, colorectal cancer and lung cancer susceptibility. Guangdong Med J 31: 2375-2377, 2010 (In Chinese).

76. Zhu Q, Jin Z, Yuan Y, Lu Q, Ge D and Zong M: Impact of MTHFR gene C677T polymorphism on Bcl-2 gene methylation and protein expression in colorectal cancer. Scand J Gastroenterol 46: 436-445, 2011.

77. Le Marchand L, Wilkens LR, Kolonel LN and Henderson BE: The MTHFR C677T polymorphism and colorectal cancer: the multiethnic cohort study. Cancer Epidemiol Biomarkers Prev 14: 1198-1203, 2005 